\title{
Uma busca por evidências do asset growth effect no Ibovespa: um estudo exploratório
}

\section{Searching for evidence of the asset growth effect in Ibovespa: an exploratory study}

Fernanda Vieira Fernandes Ribeiro*

Mestranda em Administração de Empresas pela Escola de Administração de Empresas de São Paulo da Fundação Getúlio Vargas e Analista do Banco Central do Brasil * E-mail: fernanda.vfribeiro@gmail.com

Recebido em 15.06 .2010 * Aceito em 20.07.2010 * $2^{a}$ versão aceita em 17.11 .2010

\section{RESUMO}

O objetivo deste trabalho é analisar, para o Brasil, a existência ou não de uma questão intrigante na literatura internacional: empresas com menor crescimento no ativo total observaram retornos superiores em relação àquelas com maior crescimento. Para tanto, foi considerado o Ibovespa como proxy do mercado brasileiro e, dentre as empresas que compõem o índice, foram selecionadas as não financeiras, cujos dados estivessem disponíveis no período de análise, compreendido entre 2000 e 2009. Com o objetivo de verificar se há relação negativa entre $\mathrm{o}$ crescimento dos ativos totais e os retornos das respectivas ações, primeiramente elas foram separadas em quatro carteiras, com base no crescimento médio ponderado do ativo total e, ainda, foram calculados os retornos logarítmicos. Além da estatística descritiva, foram desenvolvidos: matriz de correlações, teste $\mathrm{ADF}$ de raiz unitária, regressões lineares anuais e dados em painel. De posse dos resultados, concluiu-se que não é possível afirmar que essa relação existe para o Brasil, ao contrário do observado em outros mercados, uma vez que os testes não se mostraram estatisticamente significantes. Uma possível justificativa abordada é o fato de o Brasil possuir peculiaridades que fazem com que o mercado reaja, inclusive positivamente, ante o investimento em ativos totais, como a existência de linhas de financiamento nos organismos oficiais, permitindo que grandes conglomerados captem recursos a um custo reduzido.

Palavras-chave: Relação negativa. Crescimento de ativos. Retorno das ações. Dados em painel.

\section{ABSTRACT}

The main objective of this study is to analyze, for Brazil, the existence of an intriguing question in the international literature: firms with lower growth in total assets observed superior returns compared to those with higher growth. Therefore, the Bovespa index was considered as a proxy for the Brazilian market. Among the companies in the index, non-financial firms were selected, for which data were available for the period from 2000 to 2009. In order to verify whether there is a negative relationship between total assets growth and. stock returns, we first separated them into four portfolios, based on the weighted average growth of total assets, and the logarithmic returns were calculated. In addition to descriptive statistics, a correlation matrix, $A D F$ unit root test, linear regressions and annual panel data were developed. Based on the results, we concluded that it is not possible to say that this relationship exists in Brazil as the tests were not statistically significant. One possible reason for these results is that Brazil has lines of funding from official bodies, with low interest rates, which influences the market to react even positively in view of a growth in total assets.

Keywords: Negative relationship. Asset growth. Asset return. Panel data.

*As opiniões expressas neste trabalho são exclusivamente da autora e não refletem, necessariamente; a visão do Banco Central do Brasil. 


\section{INTRODUÇÃO}

Nas últimas décadas diversos artigos abordaram uma questão um tanto intrigante: empresas com menor crescimento no ativo total apresentaram retornos superiores em relação àquelas com maior crescimento. Essa relação negativa entre ativos totais e retornos das ações já foi alvo de estudos em diversos países, mas a literatura brasileira ainda é escassa no assunto.

Dessa forma, tendo por base estudos anteriores, como o de Fairfield, Whisenant e Yohn (2003), Titman, Wei e Xie (2004), Broussard, Michayluk e Neely (2005), Cooper, Gulen e Schill (2009), Nagano, Merlo e Silva (2003) e Galdi e Lopes (2010), este trabalho tem por objetivo verificar se há relação negativa entre o crescimento do ativo total das empresas e o retorno das suas ações no mercado brasileiro. Para tanto, a amostra considerada inicialmente compreende 26 ações listadas no Ibovespa, em um período de dez anos, de 2000 a 2009.

O estudo está organizado em seis seções: a primeira apresenta uma revisão da literatura nacional e internacional sobre o assunto; a segunda mostra a metodologia de trabalho, detalhando as formas de mensuração mediante regressão e painel; a terceira apresenta a amostra e os dados obtidos, com o ferramental da estatística descritiva; a quarta analisa os resultados obtidos, bem como os testes realizados; a quinta trata de peculiaridades do mercado brasileiro e a última conclui o estudo.

\section{REVISÃO DA LITERATURA}

O efeito do crescimento do ativo total sobre o preço das ações foi realizado por diversos autores e, em todos eles, foi observada a relação negativa entre o crescimento do ativo total e o retorno das ações, com exceção de Nagano, Merlo e Silva (2003), como apresentado a seguir.

Fairfield, Whisenant e Yohn (2003) estudaram a relação entre o crescimento do ativo operacional líquido e o retorno sobre ativos totais. A amostra utilizada foi referente a empresas cujos balanços patrimoniais e retornos estivessem disponíveis, abrangendo um período de 30 anos, entre 1964 e 1993. Utilizaram o Standard \& Poor's 2000 Compustat Industrial and Merged Industrial Research como base de dados financeiros e o Center for Research in Security Prices (CRSP) 2001 para a obtenção dos retornos mensais. Foram excluídas da amostra empresas cujos dados não seriam suficientes para calcular receita operacional, patrimônio, fluxo de caixa ou ativo líquido operacional. Isso porque uma das premissas do estudo é que assim como a rentabilidade pode se decompor em patrimônio e fluxos de caixa operacionais, o crescimento pode ser decomposto em ativos operacionais líquidos e crescimento do ativo operacional líquido no longo prazo. Por meio de regressões anuais, foi observado que o crescimento dos ativos no longo prazo e o ativo operacional líquido apresentaram relação negativa em comparação ao retorno sobre os ativos, considerando um ano de defasagem. Segundo os autores, esse resultado é consistente com as práticas contábeis conservadoras, diminuindo os retornos marginais dos investimentos.

O estudo de Titman, Wei e Xie (2004) apresenta a relação negativa entre o crescimento dos ativos totais e os benchmark-adjusted returns, sob duas perspectivas: empresas com restrição de capital e empresas com folga financeira e superinvestimento. A amostra utilizada compreende as empresas domésticas listadas na NYSE, Amex e Nasdaq, excluindo as financeiras. O período de análise foi de 1973 a 1996, com retornos mensais obtidos no CRSP e dados financeiros obtidos no Compustat. Para a análise de retornos foi utilizado o modelo characteristicbased benchmark, utilizando o modelo Fama- 
French de três fatores e o Carhart momentum factor, indicado pelos autores como o modelo de quatro fatores. Por meio de regressão verificaram que o investimento em capital possui relação negativa com o retorno e é mais forte para as empresas com maiores fluxos de caixa e menor índice de endividamento e, ainda, que esse fato se inverte quando empresas desse tipo são alvo de aquisições hostis.

O estudo de Broussard, Michayluk e Neely (2005) considerou o período de 1981 a 1990 para a formação de portfólios e de 1981 a 1995 para a análise do crescimento. Utilizando o Compustat como base de dados, foram excluídas empresas cujos dados não estivessem inteiramente disponíveis. A amostra obtida foi segmentada em quantis, classificadas por indicadores como book to market e crescimento, utilizando, ainda, na análise o conceito de Compound Annual Growth Rate (CAGR). Por meio de regressão linear, concluíram que "empresas de crescimento lento têm retornos mais elevados em relação às de crescimento acelerado basicamente por dois motivos, a saber: (1) seu crescimento quando comparado com seu passado - de crescimento lento se mostra acelerado; (2) seus ativos foram marcados para baixo, refletindo a crença de que seu crescimento futuro seria lento. Empresas que demonstram elevados crescimentos no passado também estimulam a entrada de concorrentes no seu setor o que reduz o valor de seus papeis no futuro" (BROUSSARD; MICHAYLUK; NEELY, 2005, p. 10).

Cooper, Gulen e Schill (2007), que denominam essa relação negativa como "asset growth effect", analisaram o efeito do investimento em ativos nas empresas por meio de um cross-section entre crescimento dos ativos e consequente retorno das ações. Para tanto, utilizaram uma amostra de ações de empresas norte-americanas não financeiras listadas no NYSE, Amex e NASDAQ, no período de 1963 a 2003, utilizando CRSP e Compustat como base de dados. Comparando o crescimento no ativo com diversos determinantes do retorno, encontraram que empresas com menor crescimento no ativo obtiveram um retorno anual ajustado ao risco de $9,1 \%$, enquanto as de maior crescimento, uma queda de $10,4 \%$ no retorno de suas ações. Mediante seus testes, observaram que o crescimento dos ativos é o que melhor explica os retornos futuros, visto que pelo teste-t, o crescimento é mais significativo do que indicadores como book-to-market equity, capitalização e momentum. Seu maior poder de predição é devido ao fato de que capta o impacto no retorno por intermédio dos componentes de investimento e financiamento da empresa, variando de acordo com o porte de cada uma, apesar de ser um resultado relevante, não é explicada pelo estudo, sugerindo que seja abordada no futuro.

Em seu mais novo trabalho, Cooper, Gulen e Schill (2009), revisando seu estudo anterior de 2007, realizaram um dos estudos mais recentes relacionados ao tema, verificando uma relação negativa entre o crescimento dos ativos totais e o retorno das ações. Assim como no estudo anterior, utilizaram uma amostra de ações de empresas norte-americanas não financeiras, listadas no NYSE, Amex e NASDAQ. O período de análise foi de 1968 a 2007, utilizando CRSP e Compustat como base de dados. Relacionaram o valor total do ativo das empresas publicado em dezembro e, quando não disponível, em março, com portfólios formados no mês de junho. Analisaram em períodos de um e de cinco anos e encontraram a mesma relação negativa pretendida inicialmente. A conclusão do trabalho é que, ao longo de 40 anos, o baixo crescimento no ativo total gerou um prêmio pelo retorno médio $20 \%$ superior ao ano em relação ao observado nas empresas com maior crescimento no ativo total.

Apesar de a literatura brasileira ser ainda escassa e incipiente, há estudos pertinentes ao tema que, apesar de não abordarem de forma direta a relação negativa entre o 
crescimento do ativo total e o retorno das ações, contribuem para este estudo. Nagano, Merlo e Silva (2003) estudaram o impacto de variáveis fundamentalistas sobre o retorno das ações no mercado brasileiro, verificando se o comportamento observado correspondia inteiramente ao Capital Asset Pricing Model proposto por Sharpe (1964) ou se sofria influência de outras variáveis. Dessa forma, analisaram o impacto no retorno das ações com base nas seguintes variáveis: beta, valor de mercado, vendas/ preço, fluxo de caixa/ preço, valor patrimonial/ preço, dividendos/ preço, lucro/ preço e endividamento (ativo total/ valor de mercado e ativo total/ valor patrimonial). Os autores utilizaram uma amostra composta pela totalidade das empresas não financeiras listadas no Ibovespa no período de maio de 1995 a maio de 2000 e aplicaram os dados a modelos de regressão múltipla, utilizando ainda cross-section. Como resultados relevantes para o estudo, destaque-se que as variáveis ativo total/patrimônio líquido e ativo total/valor de mercado apresentaram muitos outliers e que o indicador ativo total/patrimônio líquido apresentou relação negativa, mas não estatisticamente significante com os retornos. Observaram, ainda, que de acordo com a regressão múltipla, o comportamento das variáveis não é inteiramente explicado pelo CAPM. Isso porque não somente o beta apresenta relação linear com o retorno esperado dos ati- vos, mas também outras variáveis se mostraram significantes, como lucro/preço, valor de mercado, valor patrimonial/preço e liquidez.

Sabe-se que outliers carregam informações relevantes, devendo ser analisados com o devido cuidado. Como será discutido adiante (seção 5), Nagano, Merlo e Silva (2003) podem ter observado muitas disparidades na variável ativo total/valor de mercado devido ao grande investimento em ativo total realizado por alguns conglomerados por meio de linhas de financiamento em fontes oficiais de financiamento - como o BNDES -, montante que não é normalmente obtido no mercado bancário.

Galdi e Lopes (2010) testaram, no mercado brasileiro, um modelo teórico-empírico com o objetivo de mostrar que as variáveis contábeis possuem poder explicativo nos retornos das ações. Com uma amostra composta pelas empresas listadas na Bovespa, no período de 1997 a 2008, concluíram que o retorno das ações está relacionado ao lucro líquido, variação da rentabilidade, capital investido, variação da oportunidade de crescimento e taxa de desconto - não estudaram o impacto dos ativos totais, proposto neste trabalho. Entretanto, encontraram que essa relação entre medidas contábeis e retorno das ações é menos significante em comparação com estudos norte-americanos.

\section{METODOLOGIA}

Com o objetivo de verificar se há relação negativa entre o crescimento dos ativos totais e os retornos das respectivas ações, primeiramente, elas foram separadas em níveis de crescimento, como proposto por Cooper, Gulen e Schill (2009), com base no crescimento médio ponderado do ativo total, obtido no balanço patrimonial não consolidado. Para o cálculo do retorno das ações foi utilizado o retorno logarítmico, considerando os preços de fechamento do último trimestre de cada ano, em moeda corrente, ajustado por proventos (dividendos, splits etc) e inflação.

Para que fosse possível comparar o crescimento do ativo total das empresas com seus respectivos retornos na Bolsa de Valores, foram, ainda, calculadas as taxas de crescimento anual ponderado (Compound Annual Growth Rate - CAGR) para os ativos, como sugerido por Broussard, Michayluk e Neely (2005), e o retorno médio geométrico. 
Com o intuito de definir quatro níveis de crescimento tanto dos ativos totais, quanto dos retornos, as 26 ações foram divididas em quatro carteiras, correspondendo cada uma a aproximadamente $25 \%$ da amostra, visto que a primeira e a última seções possuem sete empresas e, as divisões intermediárias, seis empresas cada uma. Tal segmentação foi feita com base no CAGR dos ativos. Dessa forma, será possível, após a análise dos dados, verificar a relação existente entre o maior/menor crescimento dos ativos totais e o maior/menor retorno das respectivas ações das empresas analisadas.

Para verificar a relação existente entre o crescimento dos ativos totais e os retornos das ações das empresas compreendidas na amostra, foi utilizado um modelo de regressão linear simples por mínimos quadrados, utilizando dados anuais referentes ao último trimestre de cada ano.

$\mathrm{Y}=\beta_{0}+\beta_{1}(\mathrm{X})+\varepsilon$

em que:

Y: Retornos das ações;

$\mathrm{X}$ : Crescimento dos ativos totais.
Foram feitas dez regressões anuais (2000 a 2009), relacionando o crescimento dos ativos totais e o retorno das respectivas ações. Além disso, foi elaborada uma matriz de correlações, buscando complementar o modelo de regressão.

Além das regressões, foi realizado, também, um estudo com dados de painel balanceado, com efeito fixo, buscando relacionar, ano a ano, o crescimento do ativo total e dos retornos logarítmicos das respectivas ações.

Como forma de complementar o estudo, foram realizadas mais dez regressões anuais, analisando e excluindo possíveis outliers, uma vez que Nagano, Merlo e Silva (2003) encontraram muitas disparidades nas variáveis ativo total/patrimônio líquido e ativo total/valor de mercado. Foram observados, ao longo do tempo, o crescimento dos ativos e o respectivo retorno das ações, comparando tais dados com os obtidos pelas demais empresas. Após identificar prováveis outliers, foram analisadas, detalhadamente, as respectivas instituições, caso a caso. Por meio de relatórios anuais e demonstrações financeiras trimestrais, foi possível compreender os motivos para tamanha disparidade.

\section{AMOSTRA E DADOS}

Com o objetivo de verificar se há relação negativa entre o crescimento do ativo total das empresas e o retorno das suas ações no mercado brasileiro, foram selecionadas as empresas não financeiras incluídas no Ibovespa. A escolha desse índice deve-se ao fato de que as ações que o compõem são líquidas o suficiente para que outros dados - como balanços patrimoniais, a serem utilizados neste estudo - estivessem disponíveis para o período analisado. A amostra foi definida com base no Ibovespa em março de 2010, a partir das 62 ações que o compõem. Foram excluídas empresas cujas demonstrações não estivessem disponíveis para todas as datas na base de dados, totalizando 36 empresas excluídas e restando
26 empresas, consideradas como a amostra final. Para obter os dados referentes ao ativo total e preço de fechamento trimestral das ações do Ibovespa foi utilizada a base de dados do Economática referente ao final de 2000 a 2009.

Após a coleta dos dados e feitas as exclusões mencionadas, elas foram organizados conforme as tabelas apresentadas adiante. A Tabela $1 \vartheta$ apresenta o crescimento médio ponderado (CAGR) dos ativos e dos retornos (retorno médio geométrico) de 2000 a 2009 para cada ação da amostra. A Tabela $2 \oslash$ contém a estatística descritiva para os quatro segmentos e para a amostra como um todo, referentes ao período de 2000 a 2009. 
Tabela 1 Crescimento dos ativos totais e dos retornos por ação: 2000-2009

\begin{tabular}{|c|c|c|c|}
\hline & Ação & CAGR ativos & $\begin{array}{c}\text { Retorno } \\
\text { médio } \\
\text { geométrico }\end{array}$ \\
\hline 1 & LIGT3 & $-9,50 \%$ & $-24,50 \%$ \\
\hline 2 & TNLP3 & $-2,10 \%$ & $7,40 \%$ \\
\hline 3 & TNLP4 & $-2,10 \%$ & $3,20 \%$ \\
\hline 4 & CMIG4 & $0,30 \%$ & $14,90 \%$ \\
\hline 5 & TLPP4 & $1,20 \%$ & $10,10 \%$ \\
\hline 6 & CPLE6 & $2,80 \%$ & $6,50 \%$ \\
\hline 7 & CRUZ3 & $5,00 \%$ & $25,90 \%$ \\
\hline 8 & PCAR5 & $10,20 \%$ & $6,10 \%$ \\
\hline 9 & CSNA3 & $10,40 \%$ & $29,40 \%$ \\
\hline 10 & USIM3 & $11,30 \%$ & $23,10 \%$ \\
\hline 11 & EMBR3 & $14,30 \%$ & $-4,80 \%$ \\
\hline 12 & GGBR4 & $17,30 \%$ & $26,20 \%$ \\
\hline 13 & PETR3 & $19,10 \%$ & $20,50 \%$ \\
\hline 14 & PETR4 & $19,10 \%$ & $17,60 \%$ \\
\hline 15 & VIVO4 & $19,30 \%$ & $-37,10 \%$ \\
\hline 16 & ITSA4 & $19,50 \%$ & $26,20 \%$ \\
\hline 17 & BRKM5 & $19,80 \%$ & $-24,70 \%$ \\
\hline 18 & KLBN4 & $22,20 \%$ & $5,40 \%$ \\
\hline 19 & BRTO4 & $23,30 \%$ & $5,00 \%$ \\
\hline 20 & GOAU4 & $23,70 \%$ & $28,30 \%$ \\
\hline 21 & VALE3 & $25,30 \%$ & $21,90 \%$ \\
\hline 22 & VALE5 & $25,30 \%$ & $18,90 \%$ \\
\hline 23 & TCSL3 & $30,70 \%$ & $-1,90 \%$ \\
\hline 24 & TCSL4 & $30,70 \%$ & $-9,20 \%$ \\
\hline 25 & AMBV4 & $37,30 \%$ & $24,00 \%$ \\
\hline \multirow[t]{2}{*}{26} & BRFS3 & $42,90 \%$ & $19,20 \%$ \\
\hline & Total & $17,10 \%$ & $14,40 \%$ \\
\hline
\end{tabular}

Fonte: Autoria própria, com dados de Economática e Bovespa, 2010

Tabela 2 Estatística descritiva período: 2000-2009

\begin{tabular}{l|c|c|c|c|c|c|c|c}
\hline & \multicolumn{2}{|c|}{ CAGR } & \multicolumn{2}{c|}{ Média } & \multicolumn{2}{c}{ Mediana } & \multicolumn{2}{c}{ Desvio-padrão } \\
\cline { 2 - 9 } & ativos & retornos & ativos & retornos & ativos & retornos & ativos & retornos \\
\hline Carteira 1 & $-0,40 \%$ & $9,80 \%$ & $-0,60 \%$ & $6,20 \%$ & $0,30 \%$ & $7,40 \%$ & $4,70 \%$ & $15,40 \%$ \\
\hline Carteira 2 & $16,90 \%$ & $19,90 \%$ & $13,80 \%$ & $16,80 \%$ & $12,80 \%$ & $21,80 \%$ & $3,80 \%$ & $13,30 \%$ \\
\hline Carteira 3 & $19,40 \%$ & $5,00 \%$ & $20,50 \%$ & $-1,30 \%$ & $19,70 \%$ & $5,20 \%$ & $1,80 \%$ & $24,60 \%$ \\
\hline Carteira 4 & $26,60 \%$ & $17,50 \%$ & $30,80 \%$ & $14,50 \%$ & $30,70 \%$ & $19,20 \%$ & $7,10 \%$ & $14,20 \%$ \\
\hline Total & $17,10 \%$ & $14,40 \%$ & $16,00 \%$ & $9,10 \%$ & $19,10 \%$ & $12,50 \%$ & $12,90 \%$ & $17,70 \%$ \\
\hline
\end{tabular}

Fonte: Autoria própria, com dados de Economática e Bovespa, 2010 


\section{TESTES E RESULTADOS}

Analisando a estatística descritiva, não se pode afirmar que exista relação negativa entre o crescimento dos ativos e o retorno das respectivas ações no mercado brasileiro. Dessa forma, faz-se necessário prosseguir com uma análise mais robusta, aqui proposta, em forma de matriz de correlação, regressões e dados em painel.

Considerando as correlações entre o crescimento dos ativos e o retorno das ações ano a ano (Tabela $3 \mathbf{0}$ ), pode-se verificar que não são estatisticamente significantes. Sendo assim, além de a estatística descritiva mostrar que não se pode concluir que exista a relação negativa no Brasil, a matriz de correlações corrobora esse fato.

A partir desse momento, será verificado se existe relação negativa entre o crescimento dos ativos e o retorno das respectivas ações também sob o ponto de vista das regressões. Com o intuito de realizar as regressões, primeiramente faz-se necessário verificar se as séries que compõem a amostra são ou não estacionárias; para tanto, foram realizados testes ADF de raiz unitária. A Tabela $4 \oslash$ mostra que apenas o retorno em 2008 e o crescimen- to dos ativos totais em 2006, 2009 e o total são não estacionários. Ao nível de significância de $1 \%$, todos os demais se mostraram estacionários, podendo ser utilizados como variáveis nas regressões propostas. Em seguida, foram feitas dez regressões referentes ao período de 2000 a 2009 (Tabela $5 \bullet$ ).

Como pode ser observado, somente o ano de 2008 se mostrou estatisticamente significante (ao nível de 5\%); as demais séries registraram p-valores muito elevados, indicando a não adequação do modelo. Dessa forma, nada se pode inferir a respeito da existência de uma relação negativa entre o crescimento dos ativos totais e os retornos das respectivas ações, pois o ano de 2008 foi justamente uma das séries que se mostraram não estacionárias. Buscando complementar as regressões, foi realizado um estudo com dados em painel, que, segundo Hsiao (1986), possui diversas vantagens ante aos modelos de séries temporais, visto que controla a heterogeneidade e reduz a colinearidade entre as variáveis explicativas. Entretanto, como mostra a Tabela $6 \theta$, os resultados gerados foram, igualmente, não significantes.

Tabela 3 Correlações entre crescimento de ativos totais e retorno das ações, de 2000 a 2009

\begin{tabular}{l|l|l|l|l|l|l|l|l|l|l|l}
\hline & R2000 & R2001 & R2002 & R2003 & R2004 & R2005 & R2006 & R2007 & R2008 & R2009 & RTOTAL \\
\hline A2000 & 0,2898 & & & & & & & & & & \\
\hline A2001 & & $-0,2294$ & & & & & & & & & \\
\hline A2002 & & & $-0,0940$ & & & & & & & & \\
\hline A2003 & & & & 0,1266 & & & & & & & \\
\hline A2004 & & & & $-0,0292$ & & & & & & \\
\hline A2005 & & & & & & 0,2374 & & & & & \\
\hline A2006 & & & & & & & 0,2900 & & & & \\
\hline A2007 & & & & & & & & 0,2667 & & & \\
\hline A2008 & & & & & & & & & $-0,4609$ & & \\
\hline A2009 & & & & & & & & & & $-0,0152$ & \\
\hline ATOTAL & & & & & & & & & & & 0,1635 \\
\hline
\end{tabular}

***: estatisticamente significante ao nível de 1\%; **: ao nível de 5\%; *: ao nível de $10 \%$. A coluna da esquerda refere-se ao crescimento dos ativos totais para cada ano do período considerado e para o total da amostra; a linha superior apresenta os mesmos dados, referentes aos retornos das ações.

Fonte: Autoria própria, com dados de Economática e Bovespa, 2010 
Tabela 4 Testes ADF de raiz unitária

\begin{tabular}{|c|c|c|}
\hline Variável & t-stat & Probabilidade \\
\hline A2000 & $-4.857 .109 * * *$ & 0.0007 \\
\hline A2001 & $-4.234 .442 * * *$ & 0.0030 \\
\hline A2002 & $-3.458 .162 * *$ & 0.0182 \\
\hline A2003 & $-5.314 .880 * * *$ & 0.0002 \\
\hline A2004 & $-3.022 .066 * *$ & 0.0465 \\
\hline A2005 & $-2.898 .801 *$ & 0.0597 \\
\hline A2006 & 1.099616 & 0.9959 \\
\hline A2007 & $-7.260 .640^{* * *}$ & 0.0000 \\
\hline A2008 & $-2.858 .217^{*}$ & 0.0647 \\
\hline A2009 & -1.223 .271 & 0.6478 \\
\hline ATOTAL & -0.178037 & 0.9294 \\
\hline R2000 & $-6.238 .774 * * *$ & 0.0000 \\
\hline R2001 & $-5.616 .707^{* * *}$ & 0.0001 \\
\hline R2002 & $-3.176 .226 * *$ & 0.0348 \\
\hline R2003 & $-3.920 .666 * * *$ & 0.0063 \\
\hline R2004 & $-6.327 .629 * * *$ & 0.0000 \\
\hline R2005 & $-5.537 .263 * * *$ & 0.0001 \\
\hline R2006 & $-5.178 .310^{* * *}$ & 0.0003 \\
\hline R2007 & $-4.007 .007^{* * *}$ & 0.0052 \\
\hline R2008 & -2.544 .951 & 0.1175 \\
\hline R2009 & $-4.244 .308^{* * *}$ & 0.0030 \\
\hline RTOTAL & $-6.158 .865^{* * *}$ & 0.0000 \\
\hline
\end{tabular}

***: estatisticamente significante ao nível de 1\%; **: ao nível de $5 \%$; * ao nível de $10 \%$. O primeiro grupo de variáveis refere-se ao crescimento dos ativos totais para cada ano do período considerado e para o total da amostra; o segundo grupo apresenta os mesmos dados, referentes aos retornos das ações.

Fonte: Autoria própria, com dados de Economática e Bovespa, 2010

Tabela 5 Resultados da regressão linear

\begin{tabular}{c|c|c|c|c}
\hline & Coeficiente & Desvio-padrão & t-stat & p-valor \\
\hline 2000 & 0.087939 & 0.059290 & 1.483 .199 & 0.1510 \\
\hline 2001 & -0.189954 & 0.164519 & -1.154 .600 & 0.2596 \\
\hline 2002 & -0.170707 & 0.369204 & -0.462364 & 0.6480 \\
\hline 2003 & 0.298631 & 0.477563 & 0.625324 & 0.5377 \\
\hline 2004 & -0.015841 & 0.110581 & -0.143253 & 0.8873 \\
\hline 2005 & 0.418848 & 0.349829 & 1.197 .296 & 0.2429 \\
\hline 2006 & 0.102125 & 0.068786 & 1.484 .681 & 0.1506 \\
\hline 2007 & 0.418990 & 0.309118 & 1.355 .438 & 0.1879 \\
\hline 2008 & -0.515566 & 0.202650 & $-2.544 .120 * *$ & 0.0178 \\
\hline 2009 & -0.007924 & 0.106610 & -0.074331 & 0.9414 \\
\hline Total & 0.224197 & 0.276138 & 0.811902 & 0.4248
\end{tabular}

***: estatisticamente significante ao nível de 1\%; **: ao nível de 5\%; *: ao nível de $10 \%$.

Fonte: Autoria própria, com dados de Economática e Bovespa, 2010 
Tabela 6 Resultados do estudo com dados em painel

\begin{tabular}{|c|c|c|c|c|}
\hline \multicolumn{5}{|c|}{ Dependent Variable: RETORNO } \\
\hline \multicolumn{5}{|c|}{ Method: Panel EGLS (Cross-section weights) } \\
\hline \multicolumn{5}{|c|}{ Sample: 20002009} \\
\hline \multicolumn{5}{|c|}{ Cross-sections included: 26} \\
\hline \multirow{2}{*}{\multicolumn{5}{|c|}{$\begin{array}{c}\text { Total panel (balanced) observations: } 260 \\
\text { Linear estimation after one-step weighting matrix }\end{array}$}} \\
\hline & & & & \\
\hline Variable & Coefficient & Std. Error & t-Statistic & Prob. \\
\hline $\mathrm{C}$ & 0.174511 & 0.024038 & 7.259722 & 0.0000 \\
\hline ATIVO & -0.038838 & 0.051098 & -0.760082 & 0.4480 \\
\hline \multicolumn{5}{|c|}{ Effects Specification } \\
\hline \multicolumn{5}{|c|}{ Cross-section fixed (dummy variables) } \\
\hline \multicolumn{5}{|c|}{ Weighted Statistics } \\
\hline R-squared & 0.107265 & \multicolumn{2}{|c|}{ Mean dependent var } & 0.188846 \\
\hline Adjusted R-squared & 0.007646 & \multicolumn{2}{|c|}{ S.D. dependent var } & 0.417683 \\
\hline S.E. of regression & 0.416083 & \multicolumn{2}{|c|}{ Sum squared resid } & 40.33807 \\
\hline F-statistic & 1.076756 & \multicolumn{2}{|c|}{ Durbin-Watson stat } & 2.345085 \\
\hline Prob(F-statistic) & 0.369710 & & & \\
\hline \multicolumn{5}{|c|}{ Unweighted Statistics } \\
\hline R-squared & 0.104523 & \multicolumn{2}{|c|}{ Mean dependent var } & 0.166585 \\
\hline Sum squared resid & 40.45254 & \multicolumn{2}{|c|}{ Durbin-Watson stat } & 2.329818 \\
\hline
\end{tabular}

Fonte: Autoria própria, com dados de Economática e Bovespa, 2010

Considerando, da mesma forma, o retorno logarítmico como variável dependente e o crescimento dos ativos totais como variável independente, o painel mostra que não há relação negativa, visto que, além de o $\mathrm{R}^{2}$ ser baixo, o p-valor da variável "ativo" é muito alto, o que mostra a inadequação do modelo. Dessa forma, mesmo pelo estudo em painel, não se pode afirmar que exista relação negativa entre o crescimento dos ativos totais e o retorno das respectivas ações.

Analisando mais detalhadamente o desempenho de cada empresa no período, percebe-se que a LIGHT S/A registrou uma queda de $85,8 \%$ em seu ativo total em 2006, em face do ano anterior: em 2005, seu ativo totalizava R\$10,6 bilhões; em 2006, reduziu para R\$ 1,5 bilhões. Ao mesmo tempo, suas ações apresentaram retorno negativo de $60,5 \%$ em 2005, diante de uma valorização de $40,5 \%$ no ano seguinte. Fatos relevantes, divulgados em 2005, mostram que a instituição realizou um projeto de reestruturação societária ("Projeto de Desverticalização"). No final de 2005, a companhia realizou a primeira etapa do projeto, que consistiu na transferência para a LIGHT Energia S/A de ativos e passivos relacionados à geração e transmissão de energia elétrica. Dessa forma, por ter apresentado extrema disparidade ante as demais instituições da amostra, essa empresa foi considerada um outlier e, entendido o porquê foi excluída da amostra em uma nova análise estatística deste estudo.

Considerando, agora, uma nova amostra, sem a ação LIGT3, foram realizadas mais dez regressões, nos mesmos moldes das anteriores. Os resultados mostram que há relação negativa estatisticamente significante no período compreendido entre 2005 e 2008, justamente ocasião de reestruturação da Light e antes do período de turbulência gerado pela crise financeira (Tabela $7 \bullet$ ).

Como mostrado na tabela, ao nível de $5 \%$, os anos de 2005 e 2008 apresentaramse estatisticamente significantes e, ao nível 
Tabela 7 Resultados da regressão linear após exclusão de outlier

\begin{tabular}{c|c|c|c|c}
\hline & Coeficiente & Desvio-padrão & t-stat & p-valor \\
\hline 2000 & 0,09372 & 0,061214 & 1,531019 & 0,140019 \\
\hline 2001 & $-0,233488$ & 0,156347 & $-1,493403$ & 0,149536 \\
\hline 2002 & $-0,121402$ & 0,356655 & $-0,340392$ & 0,73679 \\
\hline 2003 & 0,272069 & 0,511955 & 0,531432 & 0,600445 \\
\hline 2004 & $-0,036672$ & 0,108356 & $-0,338438$ & 0,738242 \\
\hline 2005 & 0,630401 & 0,302236 & $2,085792 * *$ & 0,048807 \\
\hline 2006 & 0,141192 & 0,073874 & $1,911264 *$ & 0,069086 \\
\hline 2007 & 0,992575 & 0,487798 & $2,034810 *$ & 0,054096 \\
\hline 2008 & $-0,504021$ & 0,208583 & $-2,416405 * *$ & 0,024418 \\
\hline 2009 & $-0,011168$ & 0,109249 & $-0,102223$ & 0,919505 \\
\hline Total & $-0,000957$ & 0,288985 & $-0,003312$ & 0,997387 \\
\hline
\end{tabular}

***: estatisticamente significante ao nível de $1 \%$; **: ao nível de $5 \%$; ${ }^{*}$ : ao nível de $10 \%$.

Fonte: Autoria própria, com dados de Economática e Bovespa, 2010

de $10 \%$, em 2006 e 2007. Entretanto, como já mencionado, 2008 apresentou raiz unitária, por isso não se deve considerar que esse ano apresenta relação negativa. Dessa forma, considerando a ação LIGT3 um outlier e excluindo-a da amostra, verifica-se uma relação negativa, mas, ainda, não suficientemente forte para afirmar que exista relação negativa no Brasil - somente em alguns períodos da amostra.

\section{PECULIARIDADES DO MERCADO BRASILEIRO}

Buscando incorporar à análise aspectos típicos do mercado brasileiro, foram, também, estudadas características particulares de financiamento no Banco Nacional de Desenvolvimento Econômico e Social (BNDES), visto que está entre as fontes oficiais mais importantes do país, permitindo que grandes conglomerados captem recursos a custo reduzido.

Para se ter uma ideia da dimensão do volume concedido pelo BNDES, no período compreendido entre janeiro de 2009 e março de 2010, houve um aporte do Tesouro Nacional de R\$ 100 bilhões ao BNDES, totalmente desembolsado nas operações de crédito realizadas pela instituição. A maior parcela dos financiamentos, cerca de $43 \%$, foi destinada a grandes projetos de investimento em empreendimentos de implantação, expansão e modernização (acima de R\$ 10 milhões). Além disso, 78\% dos recursos desembolsados beneficiaram empresas de grande porte, domi- nantes nos setores de infraestrutura, insumos básicos e bens de capital.

Não obstante o montante financiado, os juros, também, são bastante reduzidos. O custo financeiro da carteira de projetos apoiados com recursos da Lei 11.948/09 apresentou uma média ponderada do índice Remuneração BNDES de 2,25\% a.a., que corresponde à soma dos campos de Remuneração Básica do BNDES, Taxa de Risco de Crédito e Taxa de Intermediação Financeira; nas operações com taxa fixa, foi retirado o efeito da TJLP do percentual final da taxa (BNDES, 2010).

Segundo Prado (2010), o BNDES surgiu devido à dificuldade de financiar investimento de longo prazo, visto que o setor bancário, historicamente, financia capital de giro, mas não investimento. Desde o governo Juscelino Kubitschek, a instituição é responsável pelo desenvolvimento de diversos setores da economia. Em relação ao 
período mais recente, o BNDES manteve a oferta de recursos durante a crise financeira, enquanto outras instituições privadas, não. Nos Estados Unidos, a disponibilidade de recursos para fazer investimentos permanece restrita, pois os grandes bancos ainda não retomaram. Dessa forma, o banco público é um instrumento fundamental do Estado para evitar que haja crises semelhantes - e o BNDES tem esse papel.

Com o intuito de incorporar à análise quantitativa tais aspectos, foram obtidos dados referentes às operações diretas de financiamento no BNDES, realizadas pelas empresas contidas na amostra. Para mostrar o volume que esses financiamentos representam no ativo total das empresas, tomouse por base o ano de 2009, por ser o mais recente considerado neste estudo e por ser um dos períodos em que não foi observada relação negativa significante. Em 2009, os financiamentos concedidos pelo BNDES às empresas da amostra representaram $2 \%$ da soma do ativo total e, em apenas um ano, empresas como Telemar captaram recursos que representam cerca de $30 \%$ de seu ativo total (Tabela $8 \boldsymbol{O}$ ). Dessa forma, essa pode ser considerada uma das peculiaridades que

Tabela 8 Operações diretas do BNDES em relação ao ativo total (2009)

\begin{tabular}{|c|c|c|c|}
\hline Ação & Op. Diretas BNDES (R\$) & Ativo total (R\$) & BNDES/Ativo total \\
\hline LIGT3 & 556.565 .959 & 3.327 .542 .000 & $16,7 \%$ \\
\hline TNLP3 & 2.821 .424 .000 & 9.510 .663 .000 & $29,7 \%$ \\
\hline \multicolumn{4}{|l|}{ TNLP4 } \\
\hline CMIG4 & 122.000 .000 & 11.863 .049 .000 & $1,0 \%$ \\
\hline TLPP4 & - & 20.126 .960 .000 & $0,0 \%$ \\
\hline CPLE6 & 169.500 .000 & 10.148 .860 .000 & $1,7 \%$ \\
\hline CRUZ3 & - & 4.927 .068 .000 & $0,0 \%$ \\
\hline PCAR5 & - & 13.312 .760 .000 & $0,0 \%$ \\
\hline CSNA3 & 557.943 .033 & 32.454 .410 .000 & $1,7 \%$ \\
\hline USIM3 & 93.788 .987 & 24.857 .346 .000 & $0,4 \%$ \\
\hline EMBR3 & - & 13.894 .372 .000 & $0,0 \%$ \\
\hline GGBR4 & - & 19.581 .864 .000 & $0,0 \%$ \\
\hline PETR3 & 9.409 .998 .497 & 315.324 .165 .000 & $3,0 \%$ \\
\hline \multicolumn{4}{|l|}{ PETR4 } \\
\hline VIVO4 & 96.176 .640 & 13.436 .538 .000 & $0,7 \%$ \\
\hline BRKM5 & 566.415 .549 & 21.551 .933 .000 & $2,6 \%$ \\
\hline KLBN4 & 623.506 .597 & 7.958 .819 .000 & $7,8 \%$ \\
\hline BRTO4 & 623.445 .000 & 20.985 .549 .000 & $3,0 \%$ \\
\hline GOAU4 & - & 9.347 .591 .000 & $0,0 \%$ \\
\hline VALE3 & 1.303.069.074 & 159.757.929.000 & $0,8 \%$ \\
\hline \multicolumn{4}{|l|}{ VALE5 } \\
\hline TCSL3 & 200.000 .000 & 8.590 .957 .000 & $2,3 \%$ \\
\hline \multicolumn{4}{|l|}{ TCSL4 } \\
\hline AMBV4 & 710.045 .584 & 34.080 .592 .000 & $2,1 \%$ \\
\hline BRFS3 & 750.000 .000 & 18.903.971.000 & $4,0 \%$ \\
\hline Total & 18.603.878.920 & 773.942 .938 .000 & $2,4 \%$ \\
\hline
\end{tabular}

Fonte: Autoria própria, com dados de Economática e BNDES, 2010 
justificam a não evidência da relação negativa no mercado brasileiro.

Analisando as peculiaridades do mercado brasileiro, percebe-se que, diferentemente dos Estados Unidos, os grandes conglomerados captam grande parte de seus recursos no BNDES, que possui taxas reduzidas em relação ao mercado. Dessa forma, a hipótese inicial de existência de relação negativa entre crescimento dos ativos totais e o retorno da respectiva ação que compõe o Ibovespa, deve ser interpretada de forma diferente no Brasil.

Segundo os estudos internacionais mencionados, um aumento do investimento em ativo total gera uma redução nos retornos de suas ações, o que implica na não adequação de modelos de precificação baseados em risco, visto que o aporte de capital em ativo fixo também impacta significativamente e de forma negativa. Entretanto, devido ao fato de ser comum no Brasil a captação em fontes governamentais a taxas reduzidas, um aumento nos ativos não gera impacto nos retornos da mesma forma que mostraram os estudos americanos, por exemplo.

Como apresentado por Prado, os Estados Unidos não possuem fonte de financiamento como o BNDES e, dessa forma, captações no mercado geram maiores riscos aos acionistas e, consequentemente, esse fato não é bem visto pelo mercado. No Brasil, diferentemente, o BNDES é visto como uma fonte de captação vantajosa - apesar de sua burocracia -, sendo o principal instrumento de financiamento de longo prazo para a realização de investimentos em diversos setores da economia.

\section{CONCLUSÃO}

Considerando o período de 2000 a 2009, não se pode afirmar que existe relação negativa entre o crescimento dos ativos totais e os retornos das respectivas ações. Dessa forma, apesar de tal relação se mostrar fortemente presente em outros países, como apresentado por Fairfield, Whisenant e Yohn (2003), Titman, Wei e Xie (2004), Broussard, Michayluk e Neely (2005) e Cooper, Gulen e Schill (2009), no Brasil ela não é significante - somente para alguns períodos, após a exclusão de LIGT3 -, como mostrou, também, o estudo de Nagano, Merlo e Silva (2003).

Além de ter uma das maiores taxas de juros do mundo, o Brasil possui, também, linhas de financiamento em organismos oficiais, permitindo que grandes conglomerados captem recursos a um custo reduzido. Dessa forma, investimentos em ativo total no Brasil não são vistos negativamente pelos investidores, pois, muitas vezes, fazem parte de programas oficiais de incentivo, direcionados, frequentemente, pelo BNDES e com aporte legal do Tesouro Nacional, inclusive.

Embora tais conclusões sejam prove- nientes deste estudo empírico, uma das limitações deste trabalho é a escassez de dados na amostra. Isso ocorre porque há poucas empresas pertencentes ao Ibovespa com dados completos para o período analisado. O ideal seria ampliar o número de empresas em cada carteira da amostra, visto que, no presente estudo, cada subdivisão possui no máximo sete observações. Dessa forma, haveria um número maior de observações em cada carteira, sendo possível analisá-las individualmente, de acordo com o nível de crescimento dos ativos em cada uma. Uma alternativa é expandir o estudo para todas as empresas listadas na Bolsa de Valores de São Paulo.

Outra sugestão para estudos futuros é realizar uma pesquisa qualitativa - aplicando surveys-, ou um estudo de evento, com o objetivo de analisar se investidores na Bolsa de Valores reagem positivamente ou negativamente diante de um novo financiamento concedido pelas mais diversas fontes. Além disso, também, pode ser realizado um estudo complementar com dados em painel não 
balanceado, buscando evidências da relação negativa em diferentes carteiras ao longo do tempo. Poderá ser obtido, dessa forma, um resultado mais robusto e, inclusive, apresen- tar a intensidade de cada relação negativa ou até positiva - existente entre cada uma das carteiras, compostas por diferentes empresas de cada setor da economia.

\section{Referências}

BOVESPA. Composição do Índice Ibovespa, 2010. Disponível em: <http://www.bmfbovespa. com.br/indices/ResumoCarteiraTeorica. aspx ?Indice $=$ Ibovespa\&idioma $=$ pt-br $>$. Acesso em: 24 mar. 2010.

BNDES. Relatório Gerencial - Recursos da Lei 11.948/09, 2010. Disponível em: <http://www.bndes.gov. br/SiteBNDES/export/sites/default/bndes_pt/Galerias/ Arquivos/empresa/download/Relatorio_Recursos_ Financeiros_1trimestre2010.pdf $>$. Acesso ém: 31 jul. 2010.

BROUSSARD, J. P.; MICHAYLUK, D.; NEELY, W. The role of growth in long-term investment returns. Journal of Applied Business Research, n21, p. 93-104, 2005. COOPER, M. J.; GULEN, H.; SCHILL, M. J. Asset growth and the cross-section of stock returns. SSRN, AFA 2007 Chicago Meetings Paper. Disponível em: $<$ http://ssrn.com/abstract $=760967>$. Acesso em: 21 mar. 2010.

;___ _ _ _ The asset growth effect in stock returns. SSRN, Darden Business School Working Paper No. 1335524, 2009. Disponível em: <http://ssrn. com/abstract $=1335524>$. Acesso em: 03 mar. 2010 .
FAIRFIELD, P.; WHISENANT, S.; YOHN, T. Accrued earnings and growth: implications for future profitability and market mispricing. The Accounting Review, n. 78, p. 353-371, 2003.

GALDI, F. C.; LOPES, R. F. Como as variáveis contábeis explicam o retorno das ações: um estudo empírico no mercado brasileiro. In: Congresso ANPCONT, 4., 2010. HSIAO, C. Analysis of panel data. Cambridge:

Cambridge University Press, 1986.

NAGANO, M. S.; MERLO, E. M.; SILVA, M. C. As variáveis fundamentalistas e seus impactos na taxa de retorno de ações no Brasil. Revista da FAE, Curitiba, v. 6 , p. 13-28, 2003.

PRADO, L. C. Rede Brasil atual, 2010. Disponível em: <http://www.redebrasilatual.com.br/temas/economia/ para-ex-assessor-do-bndes-crise-financeira-mostroupapel-fundamental-do-banco>. Acesso em: 01 ago. 2010. SHARPE, W. Capital asset prices: a theory of market. equilibrium under conditions of risk. Journal of Finance, n. 19 , p. $425-442,1964$.

TITMAN, S.; WEI, J.; XIE, F. Capital investments and stock returns. Journal of Financial and Quantitative Analysis, n. 39, p. 677-700, 2004. 